\title{
Cross-Layer Enhancement of IEEE 802.11 MAC for Mobile Ad Hoc Networks*
}

\author{
Taekon Kim ${ }^{1}$, Hyungkeun Lee ${ }^{2}$, Jang-Yeon Lee ${ }^{3}$, and Jin-Woong Cho ${ }^{3}$ \\ ${ }^{1}$ Department of Electronics \& Information Engineering, Korea University \\ ${ }^{2}$ Department of Computer Engineering, Kwangwoon University \\ ${ }^{3}$ Korea Electronics Technology Institute, Korea \\ taekonkim@korea.ac.kr, hklee@kw.ac.kr \\ \{jylee136, chojw\} @keti.re.kr
}

\begin{abstract}
In mobile ad hoc networks, the large amount of control overheads associated with discovering and maintaining end-to-end routing path information may not be tolerable. In this paper, we present the design and simulation of a new approach for IEEE 802.11 MAC based on the multipath routing information for ad hoc networks. The routing information about multiple paths discovered in the network layer is exploited by the MAC layer in order to forward a frame over the best hop out of multiple hop choices. The performance of our approach is compared with the one of the IEEE 802.11 MAC protocol via simulation. The results show that our proposed scheme exhibits a remarkable performance improvement over the IEEE 802.11 MAC protocol in terms of packet overhead and end-to-end throughput.
\end{abstract}

Keywords: IEEE 802.11, MANET, Cross-layer enhancement.

\section{Introduction}

A mobile ad hoc network (MANET) is a collection of mobile nodes dynamically organizing themselves for communication without requiring existing infrastructure. Each node in such a network operates not only as an endpoint but also as a router that has the functionality to forward data over the next hop while maintaining the route information. In MANETs, multiple network hops may be needed for communication between two distant nodes, due to the limited range of radio transmission. Therefore, the delivery of data between two nodes is much more complex and challenging in MANETs. For successful communications in such a network, a routing protocol should deal with the typical characteristics of these networks, such as limited bandwidth, high error rate, limited power capacity and node mobility. In this paper, we address a cross-layer technique between the MAC layer and routing layer and develop a new approach of IEEE 802.11 MAC that exploits such a cross-layer interaction.

This work is supported by the ubiquitous Autonomic Computing and Network Project, the Ministry of Information and Communication (MIC) 21st Century Frontier R\&D Program in Korea, and the Research Grant of Kwangwoon University in 2006. 
In MANETs, the state of a link between two nodes is governed by the channel impairments such as the interference and fading at the receiver as well as the noise. The channel impairments could be time-varying, and significant changes in fading and interference levels may lead to a transient link failure. This link failure is often sufficient for routing and transport protocols to react, which causes operational inefficiencies. Therefore, there is a need to devise a data-forwarding mechanism that can tolerate this type of link failure at short time-scales. Furthermore, intermediate nodes shared by others may cause data transmission to defer or even fail, called link blocking. The effect of link blocking as well as link failure can be alleviated by forwarding frames via an alternative path reaching the destination. An example is shown in Figure 1. The routing protocol decides a transmission path between the nodes $A$ and $\mathrm{E}$, while the node $\mathrm{B}$ is being accessed by another node $\mathrm{F}$ and the link, A-D, is temporally broken due to the high level of fading. The transmission via the $\mathrm{B}$ or $\mathrm{D}$ node leads to transmission retries, deferring transmission, increasing delay, and wasting the bandwidth as a result. An improved approach would be choosing the next hop, A-C, on an alternate possible path, A-C-E, by the cross-layer coordination between the routing and MAC layers.

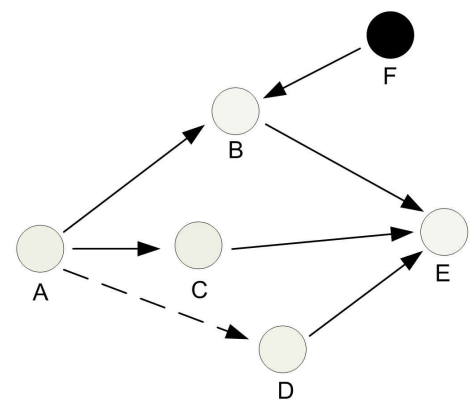

Fig. 1. Adaptive MAC protocol based on path-diversity routing

We present a new approach called anycasting [1], where multiple route information is provided to the MAC layer which is in charge of the decision on which link to forward the frame and the MAC layer must take advantage of a multiple path routing protocol. Typically, the routing protocol in the network layer decides one route out of the several paths for data forwarding, and then the MAC layer is responsible to deliver frames to the next hop along the decided route. However, let the network protocol compute multiple routing paths from the source and also from the intermediate nodes to the destination. A better approach in the MAC layer is to decide the next hop among the multiple next hops by the link status. In [2], we proposed a new dataforwarding protocol in MAC layer for vehicular ad hoc networks, where frames are forwarded to the next intermediate node without intervention of the network layer. To improve the data-forwarding performance exploiting the link status in MANETs, however, the MAC protocol requires some operational coordination between the routing and MAC layers [3]. The goal of this paper is to develop a cross-layer technique of the MAC layer, where multipath routes are discovered in the routing protocol and the virtual carrier sense mechanism is improved in the MAC protocol. 
Our routing protocol is based on the Signal Power Adaptive Fast Rerouting (SPAFAR) protocol [4] which consists of two phases namely the route discovery phase and the route maintenance phase. We modify a route discovery algorithm to find multiple paths at the source and the intermediate nodes. While such a MAC layer protocol can be designed in many ways, a proper way to design is an extension of the widely-used IEEE 802.11 [5] MAC protocol.

The remainder of this paper is organized as follows. Section 2 describes the background information such as the multi-path routing protocol based on SPAFAR and the overview of the IEEE 802.11 MAC protocol. The scheme of the proposed cross-layer enhancement of the IEEE 802.11 MAC protocol in MANETs is described in Section 3. In Section 4, the simulation results for performance comparison are shown. Finally, concluding remarks are given in Section 5.

\section{Preliminaries}

In this section, we start by briefly reviewing the multipath routing protocol to design a cross-layer enhanced protocol of IEEE 802.11 MAC based on multipath routing information. Then, the distributed coordination function (DCF) of IEEE 802.11, the MAC layer functionality, is briefly described.

\subsection{Multiple-Path Routing Protocol Based on SPAFAR}

Each node of ad hoc networks keeps a Neighbor_Table (NT) which has an updated list of its neighbors. The NT can be easily obtained by periodic broadcasts of the beacon. Each node also keeps a Routing_Table (RT) which has an updated list of all the possible routes to all the potential destinations. The RT is constructed by an on-demand routing algorithm. Each element in the RT is a five-tuple of the form <src, $d s t, n x t 1$, $c n t 1, n x t 2, c n t 2, \ldots>$. The $s r c$ and $d s t$ fields contain the unique addresses of the source and the destination node, respectively. The $n x t$ field contains the address of the neighbor node to which data packets need to be forwarded. The cnt field contains the number of intermediate nodes from the source to the destination node on this route.

The SPAFAR protocol consists of two distinct phases, the route discovery and maintenance phases. We modify the route discovery mechanism to find multiple paths from a source and intermediate nodes to a desired destination node. When a source wants to send data to a destination and its RT does not have route information to the destination, the source initiates the route discovery mechanism to find all possible paths to the destination. The route discovery mechanism is based on request-reply operations.

An $R \_$Request packet is used for the request operation from the source node and carries <src, $d s t$, rq_id, int_node, hop_cnt $>$ information. The $s r c$ and $d s t$ fields contain the addresses of the source and destination respectively. The $r q \_i d$ field contains a unique identifying number generated locally to distinguish it from the other Route Request packets. The int_node field keeps a sequence of all the intermediate nodes from the source to destination, while the packet traverses to the destination. The hop_cnt field contains the number of intermediate nodes between the source and destination. In response to a $R \_$Request packet, a $R \_$Reply packet is sent from the destination node and carries $<s r c$, dst, rq_id, int_node, hop_cnt> information. The source 
field contains the address of the node that sends the $R \_$Reply packet. The destination field contains the address of the node which sent the $R \_$Request packet. The fields, $r q \_i d$, int_node and hop_cnt, contain the packet identifier, sequence of nodes from the destination to the source and the number of hops, respectively. The int_node field in the $R \_$Reply packet is the reverse of that received in the $R \_$Request packet. The detailed operation of the routing protocol is described in [4] and [6].

\subsection{IEEE 802.11 Distributed Coordination Function}

The IEEE 802.11 MAC protocol defines two modes of operation: Distributed Coordination Function (DCF) which allows contention access for wireless media and Point Coordination function (PCF) which requires centralized access points. DCF uses a channel access mechanism known as Carrier Sense Multiple Access with Collision Avoidance (CSMA/CA). Carrier sense is performed by a combination of physical and virtual carrier sense mechanisms. A node with packets to transmit first senses the medium. If the medium is idle for at least a certain period, DIFS, it will immediately request the channel by sending a control frame, Request to Send (RTS), to the receiver node. If the receiver correctly receives RTS, it will reply with a short control frame Clear to Send (CTS). Once the transmitter receives CTS, it will start to transfer a data frame. After the successful reception of the frame, the receiver sends an ACK to the transmitter. The exchange of RTS/CTS prior to the actual data transmission reduces the collision probability in a distributed manner and copes with the hidden terminal problem [5].

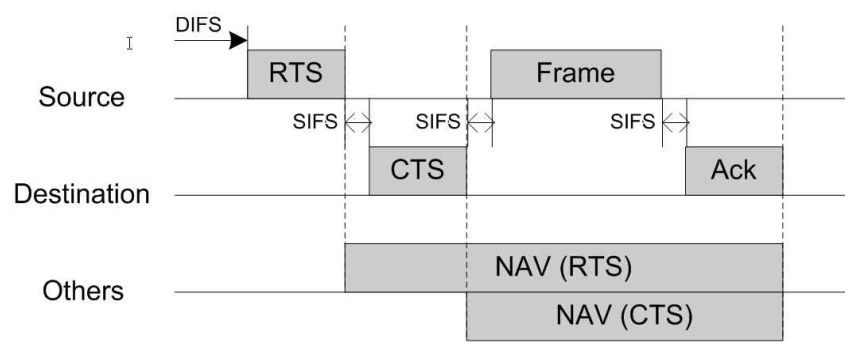

Fig. 2. Operation of IEEE 802.11 DCF

\section{Next-Hop Selection Utilizing Multi-path Information}

The MAC layer can acquire the information about possible next-hop options from the upper layer, and its responsibility is to transmit frames to any one of these receivers successfully. The modification of 802.11 DCF still uses the CSMA/CA algorithm, but takes advantage of multiple receivers to transmit the frame to any one of them. The routing protocol computes multiple routes between the source and destination. At each hop, the routing layer passes on the multiple next hop information to the MAC layer. The transmitter multicasts the RTS (MRTS) to these multiple receivers, and it 


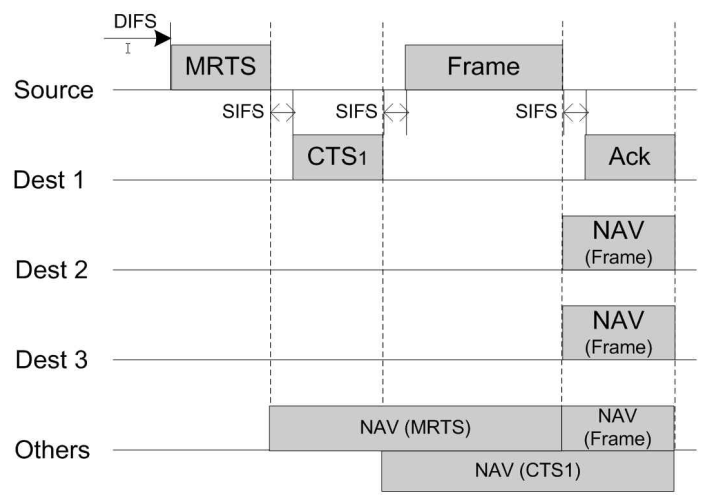

Fig. 3. First receiver's response in the cross-layer enhanced protocol of IEEE 802.11

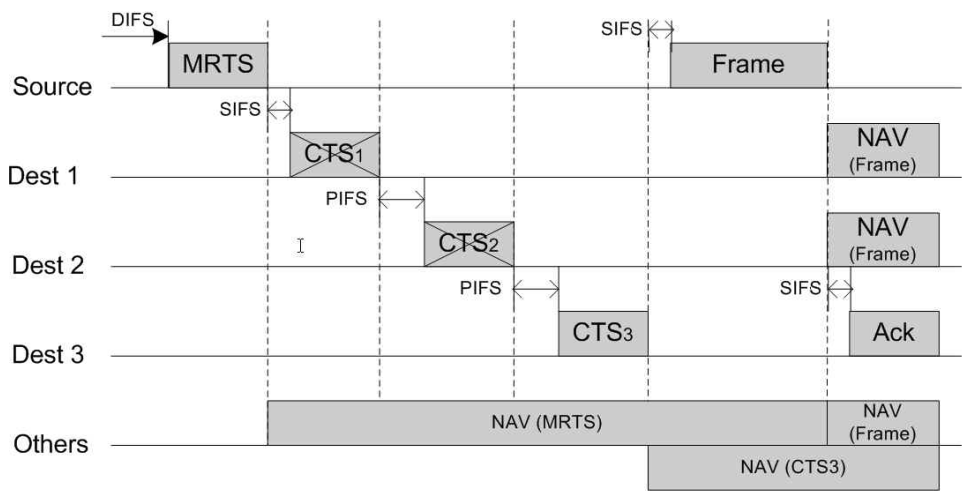

Fig. 4. Third receiver's response in the cross-layer enhanced protocol of IEEE 802.11

contains all the MAC addresses of next-hop multiple receivers. Because of practical implementation considerations, we may limit the number of next receivers to a maximum of three as shown in Figure 3 and Figure 4.

By positioning the addresses of three next receivers onto the MRTS frame, we can assign a priority order to each next hop. The priority can come from the routing or any lower layer. In the case that a shorter path to the destination gets higher priority, the routing decision in the network layer is the crucial metric for the priority. On the other hand, the information from the physical layer can be utilized to decide the priority based on the next hop that has less number of packets waiting in the queue or that has better signal strength. A combination of the above can also be used.

When the MRTS frame is broadcast to all the neighbors and all intended receivers receive the MRTS packet, the receivers respond by CTS. These CTS transmissions are intentionally delayed in time according to their priorities. The first receiver in the priority order tries to transmit the CTS after an SIFS if possible as shown in Figure 3. The second transmits the CTS after the period equal to the time to transmit a CTS, an SIFS and a PIFS if there is no transmission on the channel from the transmitter. The 
third receiver transmits the CTS after the period equal to the time to transmit a CTS, two SIFSs and two PIFSs as shown in Figure 4. When the transmitter receives the CTS from the first receiver in the priority order, the transmitter transmits the DATA frame after an SIFS interval to the sender of this CTS, as shown in Figure 3. This ensures that other, lower priority receivers hear the DATA before they send CTS and suppress any further CTS transmissions. If the first and second receivers fail to transmit the CTS and the third receiver transmits the CTS, the transmitter finally forwards the DATA frame to the third receiver as shown in Figure 4.

All the receivers hearing a CTS from any intended receiver then set their NAV until the end the ACK instance. These receivers successfully sense the carrier with the exact value of NAV. Any receiver hearing only the MRTS set the maximum NAV value with the MRTS, because the total time to deliver a DATA frame cannot be guaranteed. This duration depends upon the number of receivers (a maximum of three in this paper) to which MRTS is being sent. The value of NAV set by MRTS is updated by the DATA frame which has the exact value of NAV. Furthermore, the usage of both MRTS and CTS help any other receivers identify themselves the exposed nodes or hidden nodes, and the any other receivers hearing only the MRTS set the NAV value by the DATA frame.

If none of the CTSs are received successfully, the transmitter goes into a random backoff and then retries again with the same receivers as in IEEE 802.11. Note that the protocol reduces to IEEE 802.11 when there is only one next hop receiver, and that when multiple next hops are indeed available and the CTS from the highest priority receiver is received successfully, this would be exactly the same as IEEE 802.11.

\section{Performance Evaluation}

For the simulation, the network consisting of 25, 50, 75 and 100 mobile nodes over a fixed size $100 \mathrm{~m} \times 100 \mathrm{~m}$ terrain was considered. The maximum transmission power range is assumed to be $20 \mathrm{~m}$ between nodes. A two-state Markov model [7] is used to represent the error behavior of slowly fading channels in wireless networks. The channel of bit-error rates from $10^{-6}$ to $10^{-3}$ is applied to the above simulation environment. The traffic model uses constant bit rate (CBR) traffic along with randomly chosen source-destination pairs. A traffic rate of 1 packet/sec (512 byte packet) per flow was used in the simulation. Load is varied by varying the number of traffic.

Nodes were initially placed randomly within the fixed-size physical terrain. They move to a randomly selected destination within the terrain with a constant speed (1 meter/sec). The source and destination are selected randomly, and they also move and stop continuously in random directions during the whole simulation.

The priority is decided according to the number of hops from the source and the destination. The next hop link on the shortest path has highest priority. In order to consider the temporal changes of link status, a link is marked down and the next shortest alternative is used when the number of transmissions on the link exceeds the maximum retry count. A route error is generated only when all alternatives are exhausted. 


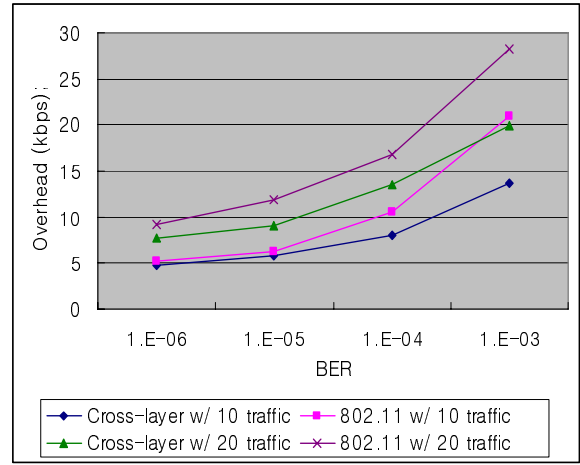

(a)

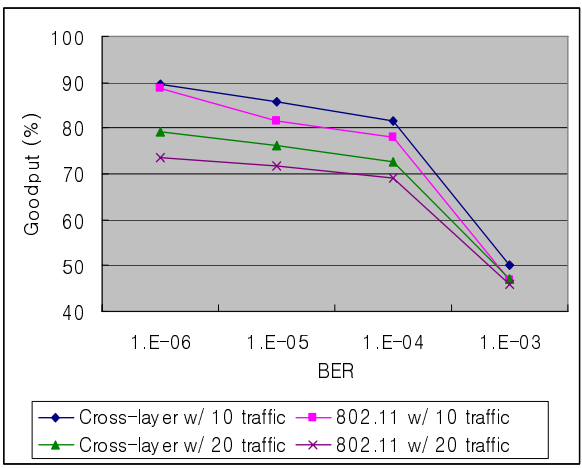

(b)

Fig. 5. Simulation Results - (a) Overhead vs. BER (b) Goodput vs. BER

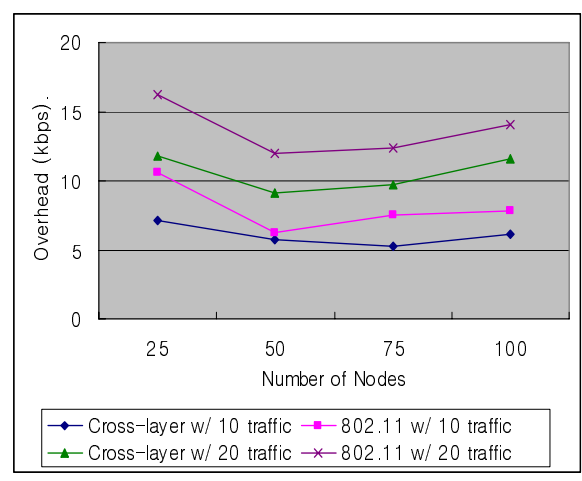

(a)

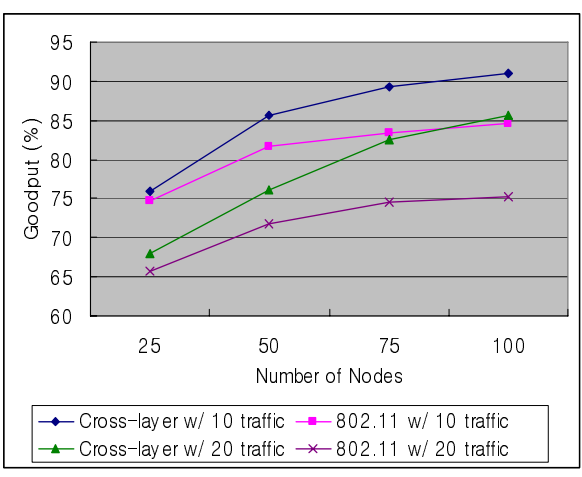

(b)

Fig. 6. Simulation Results - (a) Overhead vs. Node density (b) Goodput vs. Node density

Figure 5 and 6 represents the simulation results in terms of the overhead and goodput according to the bit-error rate and the number of nodes, respectively. In Figure 5-a, it is shown that the proposed MAC protocol based on path-diversity remarkably reduce the overhead generated by RTSs and CTSs. In particular, the improvement increases under worse channel conditions with higher bit-error-rates. Figure 5-b also represents that the proposed MAC protocol outperforms the IEEE 802.11 MAC protocol in terms of the goodput. The metric, goodput, is defined as follows; Goodput $=\frac{\text { Number of received frames in sequence }}{\text { Number of transmitted including retransmissions }}$

Furthermore, the performance improvement becomes larger with more traffic sources, that is, when the network is highly loaded. We can conjecture that pathdiversity alleviates the congested situation in the network.

Through the simulation, we can see the IEEE 802.11 MAC protocol exploiting the multipath routing information improves the network performance especially when the qualities of channels are quite low and the network is highly loaded. The performance 
improvement stems from the reduction of interactions between the MAC and network layers, and simultaneous routing deployments of multiple traffic sources.

\section{Conclusion}

We have proposed an enhanced IEEE 802.11 MAC protocol based on the cross-layer coordination to improve the performance of MANETs. The proposed protocol requires the cross-layer interaction between the MAC and network layers, and a routing protocol which discovers multiple paths from a source to the destination such as SPAFAR. The routing protocol in the network layer provides multipath information to leave the forwarding decision to the MAC layer. This cross-layer coordination increases the network performance with a reduced overhead cost.

The cross-layer enhancement of IEE 802.11 protocol we have proposed here can be applied to wireless sensor networks where the node density is quite high and node failures occur very often. Furthermore, the idea may be applied to wireless mesh networks where multipath routing information can be combined with an opportunistic routing protocol.

\section{References}

1. Choudhury, R., Vaidya, N., MAC-layer anycasting in ad hoc networks. ACM SIGCOMM Computer Communication Review, Vol. 34, 1(2004)75-80

2. Lee, W., Lee, H., Kim, K.: Packet forwarding based on reachability information for VANETs. ICOIN, 1(2007)

3. Jain, S., Das, S.: Exploiting Path Diversity in the Link Layer in Wireless Ad Hoc Networks. IEEE WoWMoM, 6(2005)22-30

4. Hwang, Y., Lee, H., Varshney, P.: An adaptive routing protocol for ad-hoc networks using multiple disjoint paths. IEEE VTC, 5(2001)2249-2253

5. IEEE Wireless LAN medium access control (MAC) and physical layer (PHY) specifications, IEEE standard 802.11-1997,(1997)

6. Lee, H., Lee, J., Cho, J.: An adaptive MAC protocol based on path-diversity routing in ad hoc networks. IEEE ICACT,2(2007)

7. Gilbert, E.N.: The Capacity of a Burst-Noise Channel. Bell System Technical Journal, 9(1960)1253-1265 